\title{
Alteraciones gasométricas en padecimientos neumológicos
}

\section{Gasometric Alterations in Pneumological Diseases}

\author{
Guevara-Cabrera $M^{1}$, De Lucio-Chavero $\mathrm{GI}^{2}$, Cervantes-Gómez $\mathrm{J}^{3}$, Huerta-Feliciano $\mathrm{E}^{4}$
}

\begin{abstract}
:
Arterial blood gas analysis is a diagnostic aid which allow us to evaluate patient's acid-base disorders, ventilatory and oxigenation abnormalities, this aid should be understood and dominated by the physician. A good interpretation can provide us a diagnostic orientation in the most representative respiratory illness.
\end{abstract}

Keywords:

Arterial blood gas analysis Respiratory illness

\section{Resumen:}

La gasometría arterial es un auxiliar diagnóstico que nos permite valorar el equilibrio ácido-base, ventilatorio y de oxigenación, siendo una herramienta de fácil alcance, debe ser dominado y entendido por el médico, la interpretación de los valores gasométricos pueden aportar una orientación diagnóstica en las enfermedades más representativas del campo de la neumología.

\section{Palabras Clave:}

Gasometría arterial, padecimientos neumológicos

La gasometría arterial es un auxiliar diagnóstico invasivo que consiste en tomar una muestra de sangre arterial, obteniendo resultados inmediatos, para analizar el equilibrio ácido-base, ventilatorio y de oxigenación de un paciente. Ofrece mediciones directas de iones hidrógeno (pH), Presión parcial de oxígeno (PaO2), Presión parcial de dióxido de carbono ( $\mathrm{PaCO} 2$ ) Bicarbonato (HCO3), lactato y Saturación arterial de oxígeno (SaO2). Útil en la evaluación de pacientes críticos o pacientes con enfermedades respiratorias, para llevar a cabo un diagnóstico, establecer un tratamiento y llevar un seguimiento de acuerdo a las necesidades de cada paciente, sus utilidades pueden ser:

* Diagnóstico de grado de lesión pulmonar Indice de Kirby (PaO2/FiO2).
* Prescripción de oxígeno suplementario con dispositivos como puntas nasales, mascarilla simple, mascarilla con bolsa reservorio, Puritan, Venturi, entre otras.

* Otorgar apoyo mecánico a través de los diferentes modos de ventilación: Controlada, Asistocontrolada, no invasiva con presión positiva, entre otros.

* Destete o retiro de ventilación.

* Existen alteraciones gasométricas en padecimientos neumológicos que son representativos como:

\section{Neumonía}

Proceso inflamatorio pulmonar de causa viral o bacteriana, en el que los alveolos tienen secreciones

\footnotetext{
${ }^{1}$ Autor de correspondencia. Universidad Autónoma del Estado de Hidalgo. Pachuca de Soto. México. Email: maryg_21_64@hotmail.com

${ }^{2}$ Universidad Autónoma del Estado de Hidalgo. Pachuca de Soto. México. Email: gvndlcchvr11@gmail.com

${ }^{3}$ Universidad Autónoma del Estado de Hidalgo. Pachuca de Soto. México. Email: judithcervantesgomez@gmail.com

${ }^{4}$ Universidad Autónoma del Estado de Hidalgo. Pachuca de Soto. México. Email: emiliohf97@ gmail.com
} 
generando consolidación, reduciendo la superficie disponible de membrana respiratoria, así como el cociente ventilación-perfusión, ocasionando así hipoxemia e hipercapnia.

\section{Asma}

Hipersensibilidad bronquial en respuesta a alérgenos que generan broncoconstricción, edema y aumento de secreciones; lo que ocasiona resistencia al flujo aéreo y disminuye la velocidad espiratoria máxima. Acidosis respiratoria.

\section{Tuberculosis pulmonar}

Enfermedad infecto contagiosa causada por el Mycobacterium tuberculosis, a nivel tisular produce una invasión por macrófagos y tabicación por tejido fibroso formado el tubérculo, cuando este proceso falla hay destrucción de tejido pulmonar con formación de grandes cavidades abscesificadas (cavernas), reduciendo la membrana respiratoria y aumentando su grosor, y con ello, una disminución progresiva de la capacidad de difusión pulmonar.

\section{Tromboembolia pulmonar}

Obstrucción parcial o completa de una arteria o varias arterias pulmonares como consecuencia de la migración de un coágulo, formado principalmente en miembros inferiores y pelvis. Aumenta el cociente VentilaciónPerfusión generando entonces un aumento del espacio muerto fisiológico. La principal alteracióne s hipoxemia hipocapnia y Saturación de Oxígeno baja.

\section{Enfermedad pulmonar obstructiva crónica (EPOC):}

Caracterizada por el atrapamiento de aire en alveolos, el cual destruye los tabiques alveolares, ocasionando resistencia de la vía aérea lo que disminuye la capacidad de difusión. Hipercapnia e Hipoxemia.

\section{Síndrome de apnea obstructiva del sueño (SAOS):}

Pausas respiratorias como consecuencia de la obstrucción parcial (hipopnea) o completa (apnea) de las vías aéreas superiores por más de 10 seg cada episodio y con frecuencia de 10 o más por hora, resultando así en una hipoxemia e hipercapnia severa.

\section{Cáncer pulmonar}

Disminución de la membrana respiratoria por la ocupación espacial del tumor, disminuyendo la capacidad de difusión. Hipoxemia e hipercapnia en grados variables.

\section{Hipertensión arterial pulmonar}

Presión de Arteria Pulmonar Media mayor de $25 \mathrm{~mm} \mathrm{Hg}$ $(3.3 \mathrm{kPa})$ y una presión capilar pulmonar menor de 15 $\mathrm{mmHg}$, este aumento de presión disminuye la capacidad de difusión generando hipoxemia, puede o no haber hipercapnia.

Por lo que es mucha utilidad conocer de qué manera se modifican los datos gasométricas en padecimientos neumológicos.

\section{Conclusión}

La gasometría es el estándar de oro para diagnosticar desequilibrios ácido-base y trastornos del intercambio gaseoso, valioso para evaluar al paciente en estado crítico así como con enfermedades respiratorias, para establecer diagnósticos, gravedad de lesión pulmonar, instituir un tratamiento de oxígeno suplementario o ventilación mecánica asistida invasiva o no invasiva.

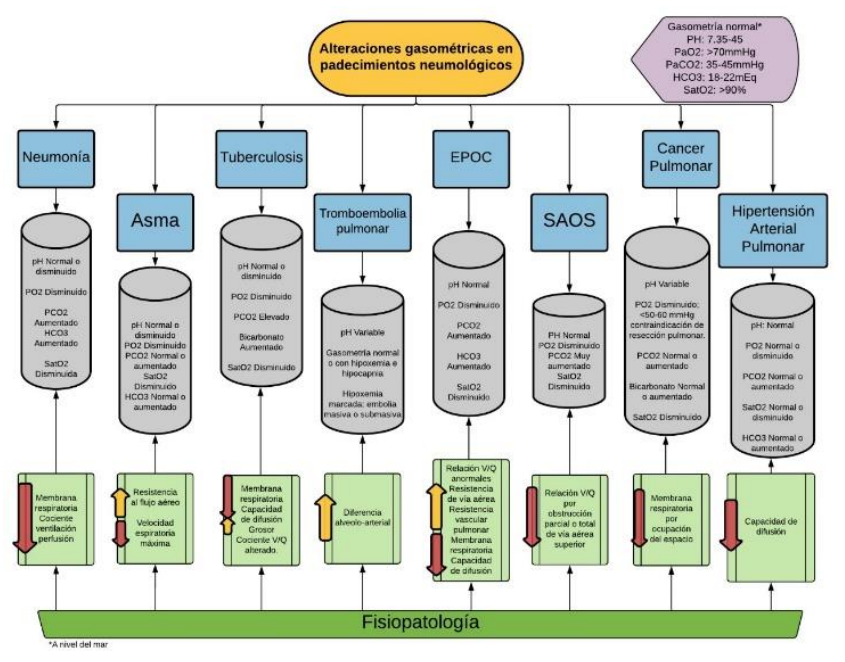

\section{Referencia}

1. Varela S., Et. Al.. (Diciembre 2005). Normativa sobre valoración del riesgo quirúrgico en el carcinoma broncogénico. Archivos de bronconeumología, 41 Número 12, 645-709.

2. Río M., Et. Al.. (2012). Actualización en el carcinoma broncogénico. Monografías Neumomadrid, 12, 130.

3. Rivero H., Et. Al.. (---). Asma Bronquial. Enfoque intensivo. Revista Cubana de Medicina Intensiva y Emergencias, 2,

4. Aramburu, C. (2016). Asma y Crisis Asmática: Diagnóstico y tratamiento. Retrieved from https://sapiensmedicus.org/asma-crisisasmatica/ 
5. Valero, A. (2018). Manejo actual de la exacerbación asmática. In --(pp. 213-214). Barcelona.

6. Jiménez C., Et. Al.. (2009). Diagnóstico y tratamiento de la crisis asmática en adultos. Neumología y cirugía de tórax, 68, s-130

7. Grupo de Trabajo sobre el diagnóstico y tratamiento de la Hipertensión Arterial Pulmonar de la Sociedad Europea de Cardiología. (2005). Guías de Práctica Clínica sobre el diagnóstico y tratamiento de la hipertensión arterial pulmonar. 15/08/18, de Revista española de cardiología Sitio web: http://www.revespcardiol.org/es/guiaspractica-clinica-sobre-el/articulo/13074846/

8. Rafael García Montesinos $\mathrm{M}^{\mathrm{a}}$ Carmen Soria Esojo . (2006). TROMBOEMBOLISMO PULMONAR: VALORACIÓN EN URGENCIAS, DIAGNÒSTICO Y TRATAMIENTO. 15/08/18, de Hospital Clínico Universitario "Virgen de la Victoria" Sitio web: http://www.medynet.com/usuarios/jraguilar/Manual\%20de\%20urgen cias\%20y\%20Emergencias/trombpul.pdf

9. Dr. Manuel J. Glez. Viñolis. (2012). GASOMETRÍA ARTERIAL. 16/08/18, de Universidad Autónoma de Madrid Sitio web: http://fuam.es/wp-content/uploads/2012/09/Tema-15-TAD-deMedicina-y-Cirug\%C3\%ADa-de-Urgencias.-Alteraciones$\%$ C3\%A1cido-base.-Gasometr\%C3\%ADa-Venosa.pdf

10. Hernández Vázquez, Julio. (2013). Tratamiento del síndrome de apnea obstructiva del sueño (SAOS) asociado a síndrome de hipoventilaciónobesidad (SHO): presión positiva continua (CPAP) versus ventilación con presión positiva BI-nivel (BIPAP). 16/08/18, de Universidad Complutense de Madrir Sitio web: https://eprints.ucm.es/17654/

11. Channick RN, Rubin LJ. Pulmonary hypertension. In: Mason RJ, Broaddus VC, Martin TR, et al, eds. Murray and Nadel's Textbook of Respiratory Medicine. 5th ed. Philadelphia, Pa: Saunders Elsevier; 2010:chap 52

12. Rich S. Pulmonary hypertension. In: Bonow ROL, Mann DL, Zipes DP, Libby P, eds. Braunwald's Heart Disease: A Textbook of Cardiovascular Medicine. 9th ed. Philadelphia, Pa: Saunders Elsevier; 2011:chap 78

13. Guyton y Hall. (2011). Tratado de fisiología médica. España: Elsevier

14. Diagnóstico y tratamiento de la hipertensión pulmonar arterial primaria en el adulto. Guía de referencia rápida. México, CENETEC; 2018 [16-08-2018]. Disponible en: http://www.cenetec.salud.gob.mx/descargas/gpc/CatalogoMaestro/43 3-

11_Hipertensixn_arterial_pulmonar/GRR_Hipertensixn_Arterial_Pul monar.pdf

15. Diagnóstico y enfermedad de la enfermedad tromboembólica venosa Guía de práctica clínica. México, CENETEC; 2018 [16-08-2018] Disponible

http://www.cenetec.salud.gob.mx/descargas/gpc/CatalogoMaestro/43 7_GPC_Enf_troboembolica_venosa/GER_Enfermedad_tromboembx lica_venosa.pdf

16. Miranda R \& Ramírez F.. (2011, Enero-Marzo). Gasometría arterial. obtención de la muestra e interpretación básica de sus resultados. Revista Médica MD , 2, 6 . 